\title{
Engineering considerations applied to starshade repointing
}

\author{
Norman Rioux ${ }^{\mathrm{a}}$, Donald Dichmann ${ }^{\mathrm{a}}$, Shawn Domagal-Goldman ${ }^{\mathrm{a}}$, Avi Mandell ${ }^{\mathrm{a}}$, Aki Roberge ${ }^{\mathrm{a}}$, Chris \\ Starke $^{\mathrm{b}}$, Eric Stoneking ${ }^{\mathrm{a}}$, Dewey Willis ${ }^{\mathrm{a}}$ \\ ${ }^{a}$ NASA Goddard Space Flight Center, Greenbelt, MD; ${ }^{b}$ Space Telescope Science Institute, \\ Baltimore, MD, USA
}

\begin{abstract}
Engineering analysis has been carried out on orbit dynamics that drive the delta-v budget for repointing a free-flying starshade occulter for viewing exoplanets with a space telescope. This analysis has application to the design of starshade spacecraft and yield calculations of observations of exoplanets using a space telescope and a starshade. Analysis was carried out to determine if there may be some advantage for the global delta-v budget if the telescope performs orbit changing delta-v maneuvers as part of the telescope-starshade alignment for observing exoplanets. Analysis of the orbit environmental forces at play found no significant advantage in having the telescope participate in delta-v maneuvers for exoplanet observation repointing. A separate analysis of starshade delta-v for repointing found that the orbit dynamics of the starshade is driven by multiple simultaneous variables that need to be considered together in order to create an effective estimate of delta-v over an exoplanet observation campaign. These include area of the starshade, dry mass of the starshade spacecraft, and propellant mass of the starshade spacecraft. Solar radiation pressure has the potential to play a dominant role in the orbit dynamics and delta-v budget. SRP effects are driven by the differences in the mass, area, and coefficients of reflectivity of the observing telescope and the starshade. The propellant budget cannot be effectively estimated without a conceptual design of a starshade spacecraft including the propulsion system. The varying propellant mass over the mission is a complexity that makes calculating the propellant budget less straightforward.
\end{abstract}

Keywords: starshade, occulter, delta-v, re-pointing, exoplanets, Hab-Ex, LUVOIR

\section{INTRODUCTION}

This paper considers factors affecting the delta-v (change in velocity) budget of a free-flying starshade occulter. The starshade flies in formation with a space telescope to provide starlight suppression for observations of exoplanets orbiting distant stars. A parameterized analysis was carried out on some of the practical aspects that affect the delta-v budget for telescope-starshade repointings. In this context "repointing" refers to changing the orbit of the starshade to align itself between the bore sight of the telescope and the next celestial target for observation. The space telescope changes its attitude while maintaining its nominal orbit to support the repointing.

\section{STAR LIGHT SUPPRESSION METHODS}

At the conceptual level, star light suppression for exoplanet observations could be carried out with a free-flying starshade and a space telescope, or with a coronagraph that is internal to the space telescope observatory. Each of these approaches has their own strengths and challenges.

A free-flying starshade tends to impose less stringent requirements on the stability of the telescope, and a starshade can be a useful tool for in-depth study of an exoplanet that has been previously identified by other means. While the starshade is changing its orbit to align with the next target for exoplanet observations, the telescope would be available for making other scientific observations without the starshade.

With regard to conducting a survey for exoplanets with a star shade, it could take on the order of weeks to re-position from one target to another due to its significant distance from the telescope. This in turn tends to drive down observing 
efficiency and exoplanet yield. Yield calculations of a starshade-based exoplanet mission need to incorporate an understanding of the delta-v budget for repointing the starshade. This paper is intended to provide some insight into some of the mission parameters that drive the delta-v budget.

An internal coronagraph repoints easily with the telescope that it is installed in. For this reason, it can be useful for exoplanet surveys and achieving a larger exoplanet yield. Internal coronagraphs tend to place more stringent requirements on telescope stability in ways that free flying starshades do not. The state of technology is dynamic. Both starshades and coronagraphs are currently the subject of technology development programs and their design and performance characteristics are progressing. It is reasonable to expect that progress in technology that will benefit both starshades and coronagraphs will continue over the next decade. Ongoing technical trade studies to optimize realizable systems will be necessary in order to ensure the most cost-effective approaches for meeting a variety of science objectives are understood.

\section{RELATIVE POINTING DINAMICS BETWEEN TELESCOPE AND STARSHADE}

This paper focuses on a telescope and free-flying starshade in Sun-Earth Second Lagrange Point orbit (L2). For a formation of two spacecraft, the question arises as to whether the global delta-v budget may be optimized by apportionment of station keeping responsibilities between the two spacecraft. To understand this question, we examined the environmental forces that dominate station keeping in the neighborhood of a libration point: differential solar radiation pressure (SRP) forces, and differential gravity ("gravity gradient", or GG) forces. If nonlinear forces are significant, then we might expect some global savings by splitting station keeping duties between the two spacecraft; for constant or linear forces, no such optimization exists.

The acceleration due to SRP force exerted on a spacecraft is directly proportional to the projected area and reflectivity, and inversely proportional to the spacecraft mass. We combine these parameters into a single ballistic coefficient (beta) for brevity. SRP acceleration is also proportional to the solar pressure itself, which is inversely proportional to the square of the distance from the Sun. Even for formations on the scale of a starshade mission (up to 100,000 km separation) the difference in solar pressure is negligible, and the differential SRP acceleration is entirely proportional to the difference in ballistic coefficients. Thus the global delta-v budget is not affected by which spacecraft compensates for SRP force.

The gravitational force near a libration point is certainly a nonlinear function of position. Using equations of motion of the circular restricted three body problem (CR3BP), and considering perturbations in the neighborhood of a JWST-like halo orbit around Sun-Earth L2, we performed a numerical study to find the magnitude and direction of the gravitygradient acceleration as a function of separation distance. It was found that the GG acceleration is dominantly linear with separation distance, and is maximized when the separation axis is approximately in the direction of the Earth.

Since the GG acceleration and the SRP acceleration are both dominantly linear, we conclude that the global delta-v budget is insensitive to apportionment of delta-v. Based on this analysis, there is apparently no advantage if the telescope performs delta-v maneuvers to contribute to the necessary telescope-starshade alignment for exoplanet observations. So for this paper, we consider the case where the telescope sustains its nominal orbit while changing its attitude to point at an exoplanet observation target, while the starshade is a free-flying spacecraft that changes its orbit to align itself between the telescope and the exoplanet observation target.

\section{NATURAL ORBIT MOTION AND FORCED DISPLACEMENT}

Moving the starshade to align with the telescope to observe a new target is accomplished by a pair of orbit maneuvers. The first maneuver is to start the relative motion of the starshade with respect to its appropriate position in relation to the telescope. The second maneuver is to stop the relative motion of the starshade with respect to its appropriate position and velocity in relation to the telescope.

The delta-v needed to start and stop the starshade does not depend on the total distance the starshade must move from one target to another, but on the distance the starshade must be moved in addition to the natural motion of its orbit. This 
distance is referred to in this paper as "forced displacement". To determine the forced displacement we need to model the orbit dynamics. A diagram of the relative motion of the starshade and telescope appears below.

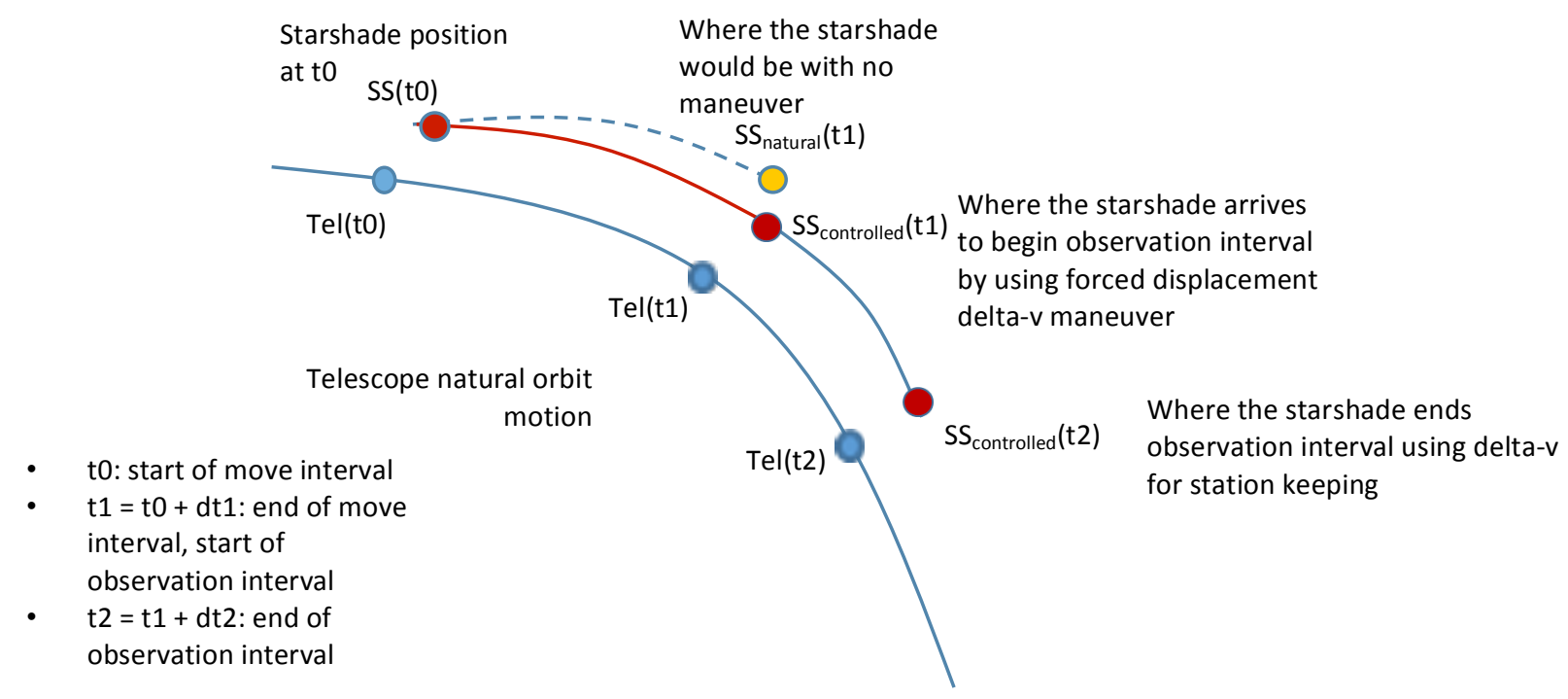

Figure 1. Natural Orbit and Forced Displacement Delta-V

\section{SCOPE OF ANALYSIS}

We conducted an analysis of parameters that drive the delta-v budget of a starshade spacecraft in a Sun-Earth L2 orbit flying tens of thousands of $\mathrm{km}$ away from a telescope. From an engineering standpoint, one would want to have a list of pointing targets for calculating the delta-v necessary for the starshade to achieve and maintain position for observing targets with the telescope. At this time, an authoritative a-priori list of desired star locations for exoplanet observations is not available. This is because the target observation sequence must be optimized to achieve the highest exoplanet yield, and this optimization requires an understanding of the delta-v required for each observation. This optimization is currently under study through modeling and simulation with design reference missions ${ }^{1-4}$.

The objective of the analysis to follow is to examine the functional dependence of delta-v on mission design parameters such as telescope-starshade distance, move interval and observation interval. A target observing set is described further on in this paper, but the only role it plays is to provide a plausible set of orbital movements for the starshade to exercise the orbit dynamics at play. Any particular target observing sequence used is not a focus of this analysis. The topic of exoplanet yields achievable with a starshade is outside the scope of this parametric analysis of starshade delta-v.

The delta-v budget and propellant used by a starshade in Sun-Earth L2 orbit will directly result from the schedule of pointing locations and observation times over the course of the mission. However, the purpose of this paper is not to predict the delta-v and propellant budgets for a starshade mission. The propellant budget derives from the delta-v budget and the design of the propulsion system for a particular starshade spacecraft. Design of starshade spacecraft and its propulsion system is not in the scope of this paper. Comments on propulsion system design considerations are provided in section 9. below.

\section{ANALYSIS APPROACH}

Though it is not the focus of this paper, having some kind of target sequence is necessary to support the parametric analysis of delta-v. To provide this capability, two kinds of target selection assumption scenarios were used as described below. Both of these scenarios assume a set of targets randomly distributed around the celestial sphere. From this distribution, a set of targets that would be viewable at the start of the observation period is determined. To be viewable 
means that the Sun-starshade-target angle is in the range of 45 degrees to 85 degrees. This angle range is specified to ensure that the sun does not reflect off the front of the starshade into the telescope.

Scenario 1: Choose a target that requires low delta-v to reach. Sort the visible targets according to their forced displacement, smallest to largest at start of the observation. Choose the target that is at the 10th percentile (i.e., only $10 \%$ have smaller displacements). Using the percentile criteria allows the number of potential targets to be increased while remaining in a regime of relatively lower delta-v.

Scenario 2: Choose a random target. This scenario disregards delta-v cost for reaching a target, so it represents a more stressing case.

Neither of these particular selection algorithms allow a target to be observed twice. This is done primarily to force the telescope to look at a wider range of targets over multiple years. It is understood that in reality it is sometimes desirable to re-examine a target several months after it is observed. Target revisit strategies are considered in exoplanet yield optimization analyses, like the ones in references 1 and 4

The analysis of each pointed exoplanet observation made by the telescope and starshade pair is divided into two intervals. There is the move interval, which is required to move the starshade to the required position to begin the observation. Then there is the observation interval (or target station keeping interval) required to perform the observation. For each exoplanet observation we select the target to observe, and we compute three maneuvers of the starshade to accomplish this observation:

dvStart: maneuver at start of move interval to drive the starshade toward its required position

dvStop: maneuver at end of move interval to match the velocity of the telescope, to begin flying at fixed displacement from telescope

dvObservation: continuous burn required for formation flight during the observation interval, to cancel the acceleration difference between starshade and telescope

At the start of the move interval, the orbits of both the telescope and the starshade are propagated to start the epoch of observation, assuming no maneuvers. Then a computation is made of the direction from the telescope to the starshade assuming natural motion. The maneuver dvStart at start of move is computed for shifting the starshade position to the desired position to observe the target. After that, the starshade motion is propagated to its projected location at the start of the observation interval. Based on this result, the dvStop necessary to allow the star shade to match the telescope velocity and begin formation flight is calculated. Having initially established formation flight, the dvObservation required to cancel the acceleration difference between telescope and starshade is computed. Then the orbits of the telescope and starshade are propagated to the end of the observation period. This represents the state of the system at the start of the next observation interval. Note that the sensing and control necessary for the starshade telescope pair to acquire and maintain precision formation flight for exoplanet observations is beyond the scope of this paper. This paper considers only the delta-v budget necessary to maintain formation flight based on idealized knowledge and control of the telescope-starshade-exoplanet target alignment.

\section{Solar Radiation Pressure (SRP) Impact on Station Keeping Delta V}

SRP can play a significant role in the delta-v budget for a starshade spacecraft. A basic parametric analysis of SRP was performed to examine the relative significance of the forces at play. To model the effects of SRP forces on the telescope, we need to know its mass, area to the sun and reflectivity coefficient $C_{-}$R. The parametric analysis in this paper is not intended to be specific to any particular telescope design. A plausible cylindrical shape telescope in Sun-Earth L2 orbit could be WFIRST, so parameters for this telescope are used. For this example, mass is specified as $8000 \mathrm{~kg}$, the area is fixed at $50 \mathrm{~m}^{2}$, and the reflectivity coefficient is chosen to be 1.5 (halfway between the extremes of 1.0 or 2.0 ). If one wanted to do a worst-case calculation, one might pick $\mathrm{C}_{-} \mathrm{R}$ values to maximize the difference in SRP between the telescope and the starshade. For the purpose of this simplified analysis, the change in area exposed to SRP as the telescope changes attitude to point to different targets was not included. In this analysis, the telescope shape was modeled as cylinder and the starshade was modeled as a flat disk with a much larger radius than the telescope. Given 
these assumptions, the change in area exposed to SRP of the starshade will be the dominant factor driving the difference in response to SRP between the telescope and the starshade.

For the telescope, using the values above, we assume

Ballistic coeff $\beta=C_{-} R *$ area $/$ mass $=0.0094$

The analysis in this paper is not intended to be specific to any particular starshade spacecraft design. But for the sake of having some numbers to plug in to the parametric analysis, the starshade from the Exo-S study report is used (reference 5). From the Exo-S report, we can assume the starshade spacecraft dry mass is about $1000 \mathrm{~kg}$, and diameter is $34 \mathrm{~m}$. The starshade mass has a significant effect on SRP. To generate representative numbers the analysis assumed a propellant mass of $500 \mathrm{~kg}$, for a total mass of $1500 \mathrm{~kg}$. The analysis in this paper is very basic, and does not include the effects of the changing mass of the starshade spacecraft as propellant is consumed. Taking the changing starshade mass into account will be best done with a detailed model of a starshade spacecraft including the propulsion system. Modelling at that level of detail is beyond the scope of this paper.

The area exposed to SRP will vary depending on the attitude of the starshade with respect to the SRP. The formulas for delta- $v$ derived for this analysis are dominated by the trigometric relationship between the area of the starshade and the direction of the SRP. For the sake of simplicity, we assume that the force on the starshade is in the same direction as the sun to starshade spacecraft direction. Thus we do not need to explicitly model dependence of delta-v on attitude. For the starshade the reflectivity coefficient is simplistically chosen to be 1.5 (halfway between the extremes of 1.0 or 2.0).

Given these simplifying assumptions, the effect of a change in attitude of the starshade is a change in area exposed to the sun.

To maintain a simple initial analysis, we model the starshade as a cylinder whose diameter is significantly larger that its width. We call the starshade diameter $\mathrm{D}$ and width $\mathrm{w}$. Let $\mathrm{S}$ be the angle from the cylinder longitudinal axis to the Sun. Then the area of the starshade exposed to the Sun is approximately

Area $=\left(\pi \mathrm{D}^{2} / 4\right) \cos (S)+D w \sin (S)=D[(\pi D / 4) \cos (S)+w \sin (S)]$
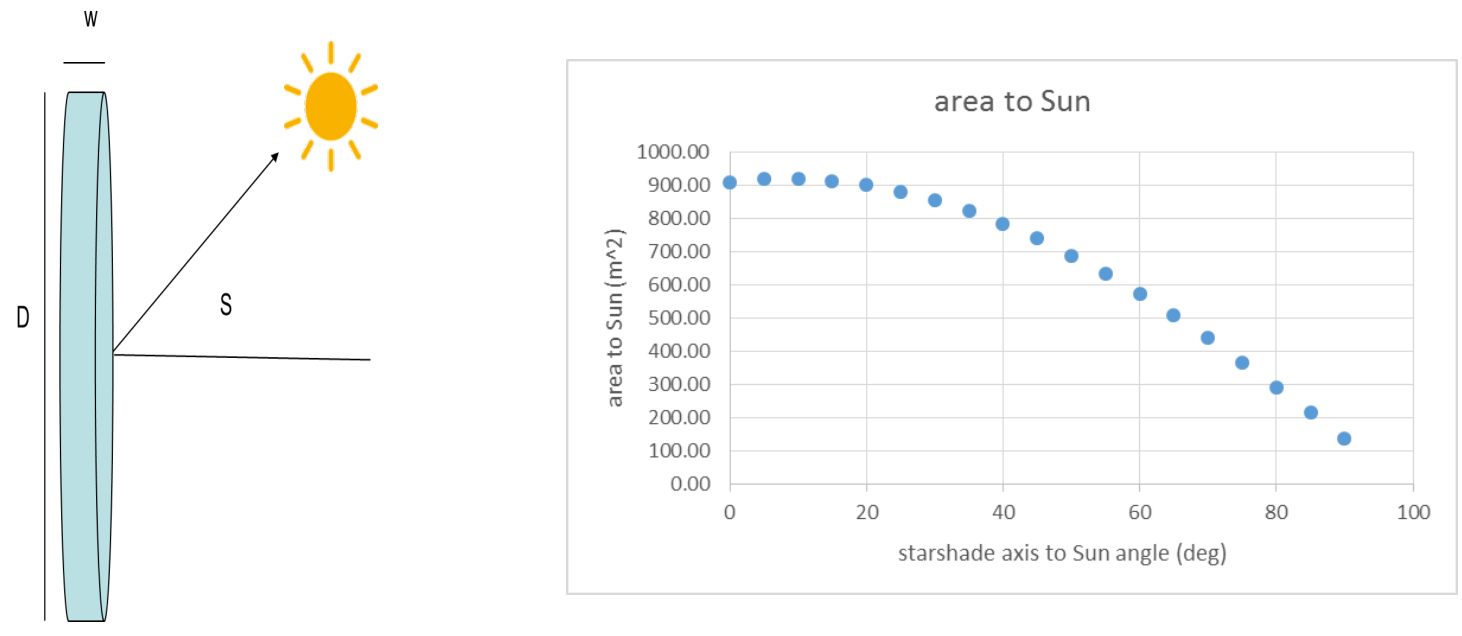

Figure 2. Area to sun vs, sun angle for $\mathrm{D}=34 \mathrm{~m}, \mathrm{w}=4 \mathrm{~m}$

Since we do not know the SRP coefficients for the telescope and the starshade accurately, we model delta- $\mathrm{V}$ with the SRP ballistic coefficient difference $\Delta \beta=\left|\beta_{\text {telescope }}-\beta_{\text {starshade }}\right|$ as a parameter. 
We make a set of simplifying assumptions for carrying out the orbit dynamics analysis. For the simple parametric analysis of this paper, none of these assumptions are expected to drive the results.

- The Earth moves in a circular orbit around the Sun

- $\quad$ Moon gravitational perturbations are neglected

- SRP is modeled as described in the preceding text

- The telescope follows an idealized halo orbit with y-amplitude $800,000 \mathrm{~km}$ and z-amplitude $500,000 \mathrm{~km}$.

To estimate the delta-v required to maintain pointing formation during the observation, the acceleration difference at the start of the observation interval is computed and it is assumed to be constant throughout the same observation interval. For this simulation we assume the starshade is initially in the same halo orbit as the telescope, a distance D ahead (about 1.4 days if $\mathrm{D}=70,000 \mathrm{~km}$ ). If we did not maneuver the starshade, it would stay in formation with the telescope.

The simulation starts the telescope near the northernmost point, where the orbit is farthest from the Earth and Sun. However, the total delta-v does not appear to depend strongly on the location of the telescope in its orbit. This is because the analysis modeled more than one year of time, which took the starshade all around the orbit.

A series of simulations was run of the orbit dynamics of the starshade telescope pair, based on the parameters and relationships described above. By fitting curves to the simulation results, approximations for the delta-v of the starshade observing sequence were developed. The values for the $\mathrm{S}$ and $\mathrm{C}$ terms in the formulas below were developed through the curve fitting process.

Based on the previous discussion,

dvMove is approximated with the following formula

$$
\begin{aligned}
\text { dvMove }(\mathrm{km} / \mathrm{sec}) & =\boldsymbol{S}_{\text {move }} * \boldsymbol{C}_{\text {move }} *(\text { forced displacement in } \mathrm{km}) /(\text { move interval in days }) \text { where } \\
S_{\text {move }} & =1+10|\Delta \beta| \\
C_{\text {move }} & =2.5 e^{-5}
\end{aligned}
$$

The $\mathrm{S}_{\text {move }}$ term represents the SRP dependence aspect of the slew

dVObservation is approximated with the following formula

$$
\begin{aligned}
& \text { dvObservation }(\mathrm{km} / \mathrm{sec})=\boldsymbol{S}_{\text {observation }} * \boldsymbol{C}_{\text {observation }} *(\text { telescope-occulter distance in } \mathrm{km}) *(\text { observation interval in } \\
& \text { days) } \\
& \begin{aligned}
S_{\text {observation }} & =1+259|\Delta \beta| \\
C_{\text {observation }} & =3.8 e^{-9}
\end{aligned}
\end{aligned}
$$

The $\mathrm{S}_{\text {observation }}$ term represents the SRP dependence aspect of the observation delta-v

dvTotal $=$ dvMove + dvObservation

\section{Conclusion with Respect to SRP}

The total delta-v (dvTotal), and the delta-v associated with the observation period (dvObservation) in particular, depend strongly on difference in SRP ballistic coefficients $|\Delta \beta|$. Thus to reduce total delta-v it is important to keep $|\Delta \beta|$ as small as possible.

However $|\Delta \beta|$ depends on the starshade area exposed to the sun and starshade mass, which change as the attitude changes and as propellant is used. Thus it may not be feasible to keep $|\Delta \beta|$ close to 0 , or even close to a constant value. Future 
starshade spacecraft design studies should examine if it is feasible to design the system so that $|\Delta \beta|$ is acceptable for a range of attitudes and masses. Figure 3 provides an example of $|\Delta \beta|$ (absolute value of delta beta) changing over a range of starshade axis to sun angles.

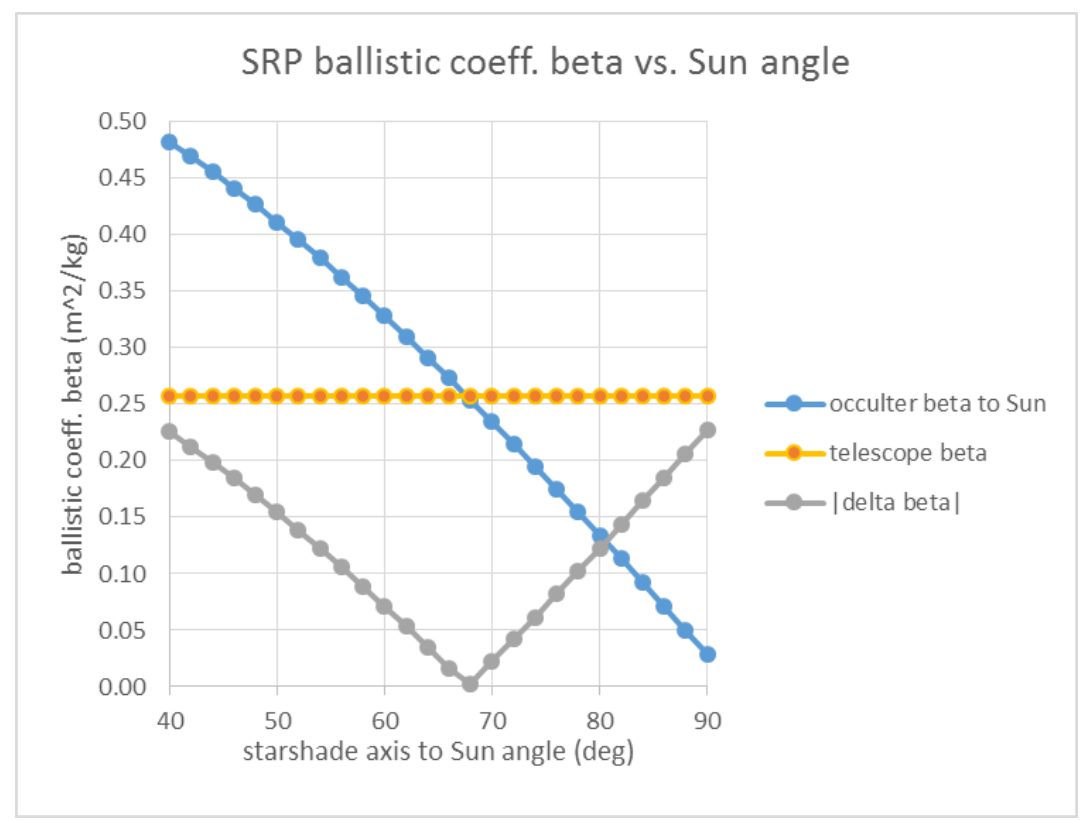

Figure 3. Graph of SRP Ballistic Coefficient (beta) Versus Sun Angle

\section{PROPULSION SYSTEM CONSIDERATIONS}

The design of the starshade spacecraft and the propulsion system play a significant role in determining the propellant budget. The propellant budget drives the mass of the propellant, which has the potential to become a significant portion of the starshade spacecraft mass. The propellant mass changes through the course of the starshade exoplanet observation mission. If the change in propellant mass is significant enough, it may play a role changing the ballistic coefficient of the starshade over the course of the mission, which will change the effect of SRP on the delta-v budget and on the propellant budget. These interdependencies make it impractical to estimate the starshade propellant budget without declaring a particular starshade spacecraft design for evaluation.

An obvious trade study with regard to the propulsion system of a starshade spacecraft is the relative benefits and drawbacks of chemical versus ion-electric propulsion. This trade study requires the specification a particular star shade mission for analysis, including an understanding of the design of the associated space telescope. It is clear that the electric power requirements for a proposed ion-electric propulsion system will need to be understood at the parametric level, so that an assessment of the necessary solar array area can be made. If the starshade must rotate as part of the mission design, this will need to be accounted for in how electric power will be provided to the ion propulsion system. An understanding of what effects station keeping thrust plume(s) may or may not have on the exoplanet observations will also need to be accounted for. Depending on the mission characteristics, it may be that a feasible system could incorporate a well-balanced design that includes chemical and electric ion propulsion. A detailed trade study could examine this question.

\section{MANAGING SRP WITHOUT PROPULSION}

One may wonder what are the alternatives to managing SRP with a propulsion system. It does not immediately appear that there is an easy and obvious alternative that alleviates the need for using propulsion for managing SRP. One could conceive of a deformable solar sail, but there would be a number of complexities that would need to be overcome. The 
sail would need to be deployable, and analysis would be needed to determine its size. The area of the sail would need to have some degree of variability, and that would need to be quantified. The variation in propellant mass over the course of the mission would also need to be taken into account while analyzing the degree of variation in the solar sail area necessary. The deployment and deformability features would add additional failure modes and some degree of additional risk to the starshade mission. The deployed configuration and variation in the area of the solar sail would need to be prevented from interfering with the starlight suppression performance of the starshade when viewed from the telescope during exoplanet observations. The modal frequencies of the deformable sail world need to be specified and designed for compatibility with the starshade. The mass would need to be kept low.

The relative technical maturity and mass of a propulsion system and its propellant could provide a bounding case for comparison of feasibility of a deformable solar sail approach. An interesting break point for analysis might be determining how long does a mission have to be in duration before the propellant mass used in station keeping is more than the mass of a deformable solar sail sufficient to equalize ballistic coefficients between the starshade and its associated telescope.

A practical alternative to a solar sail that can adjust its area dynamically during the mission would be a fixed solar sail. The fixed solar sail would not attempt to alleviate the differences in the effect of SRP between the starshade and the telescope. Its purpose would be to bias the area of the starshield exposed to SRP so that over the course of the mission and the variation in the propellant mass and expected variations of angle of the starshade with respect to the SRP, the overall delta-v budget is minimized. This would require a comprehensive analysis of all the variables at play and the science operations profile, but could be performed with an appropriate documentation of assumptions and liens against the analysis at this relatively early stage of starshade mission development.

A more exotic approach than a solar sail might look into the degree to which the reflectivity of the surfaces of the stray light shield (and perhaps the telescope as well) could be dynamically varied on orbit, and how much benefit that variation could offer to managing SRP. This is a capability beyond the mainstream of mature spacecraft technologies and may involve a technology development program.

\section{SIMULATIONS ILLUSTRATING PARAMETERS AFFECTING DELTA-V}

On the next pages, figures 4 through 11 illustrate parameters that affect delta-v for a space telescope and free-flying starshade occulter. In these figures, the parameter $\mathrm{D}$ is the starshade diameter, dtMove is the time allotted to moving the starshade to its new observing position, and dtWatch is the time spent stationkeeping while observing the exoplanet target. The dtWatch parameter corresponds to the time associated with the dvObservation maneuver discussed above. Each parameter set of D, dtMove, and dtWatch is represented with a group of three graphs and with a text box that comments on the effect of the parameters on delta-v.

A comparison between the figures provides a view into the effect of changing a parameter on the mission delta-v. A comparison of figures 4 and 5 shows the effect of increasing the D parameter (distance between telescope and starshade). A comparison of figures 5 and 6 shows the effect of decreasing the dtWatch parameter. A comparison of figures 5 and 7 shows the effect of increasing dtMove parameter. A comparison of figures 7 and 8 shows the effect of decreasing the D parameter. Figure 9 shows the effect of selecting target randomly instead of target requiring 10 th percentile delta-v as in Figure 7.

As discussed in the sections above, the effect of the SRP and the difference in ballistic coefficients of the starshade and the telescope $(|\Delta \beta|)$ can have a large effect on the delta-v budget. For figures 4 through 9 , the simulations have been configured so that the difference in ballistic coefficient between the telescope and the starshade is zero. This is for the purpose of more clearly illustrating the effects of the $\mathrm{D}$, dtMove and dtWatch parameters.

In figures 10 and 11 the simulation has been configured so that there is a difference between the ballistic coefficients of the telescope and the starshade. In this case, the difference in the ballistic coefficients was configured by changing the angle of the starshade exposed to the SRP from a value where the ballistic coefficients were roughly in balance, to an angle where there is a significant difference in the ballistic coefficients. For simplicity, this simulation did not include 
the effect of changing propellant mass during the mission. Figures 10 and 11 illustrate the potentially large effect that differences in the ballistic coefficients can have on the delta-v budget. A more robust simulation would be needed to draw quantitative conclusions for any particular telescope-starshade pair. The simulation would need to specify conceptual design properties such as the area and reflectivity coefficients of the telescope and starshade, as well as mass of the starshade and telescope. A more detailed simulation should also address the variation in starshade mass with propellant consumption. This would require a conceptual design for the design of the starshade propulsion system. 


\section{$D=35,000 \mathrm{~km}$, dtMove $=7$ days, dtWatch $=14$ days}

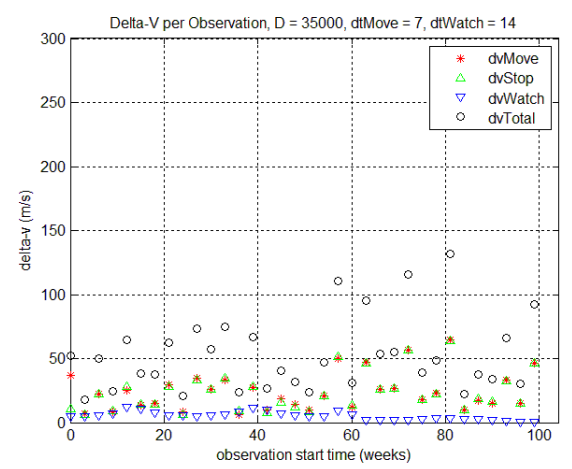

- Top left plot shows the delta-v cost for each observation, over a 2-year mission

- Delta-v increases slightly in 2 year because the targets easiest to reach have already been observed

- Bottom left shows the cumulative delta$v$ as the mission progresses

- Bottom right shows delta-v for each observation, as a function of the distance the occulter must be shifted.
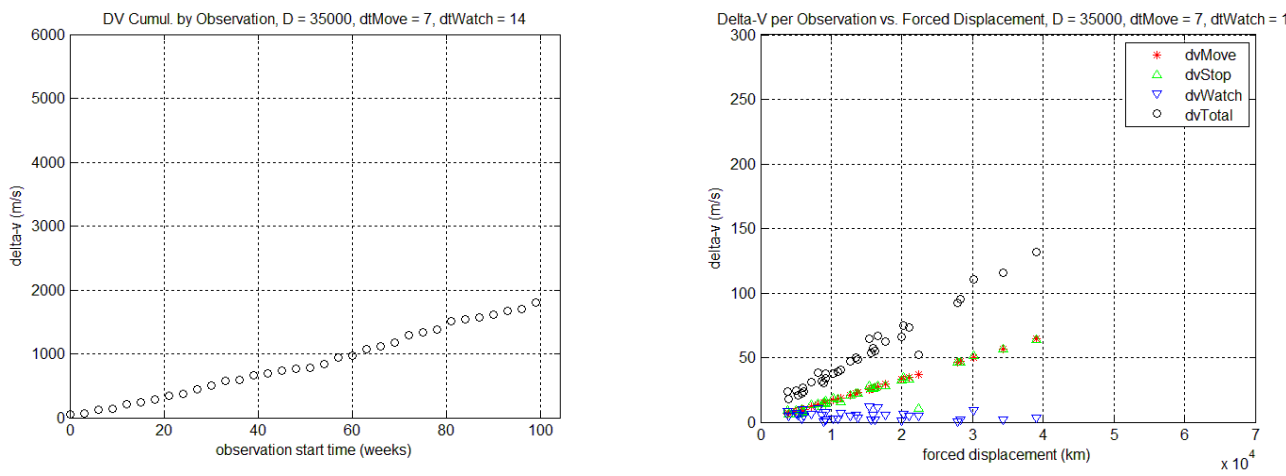

Figure 4. Parameters set at $D=35,000 \mathrm{~km}, \mathrm{dtMove}=7$ days, $\mathrm{dtWatch}=14$ days

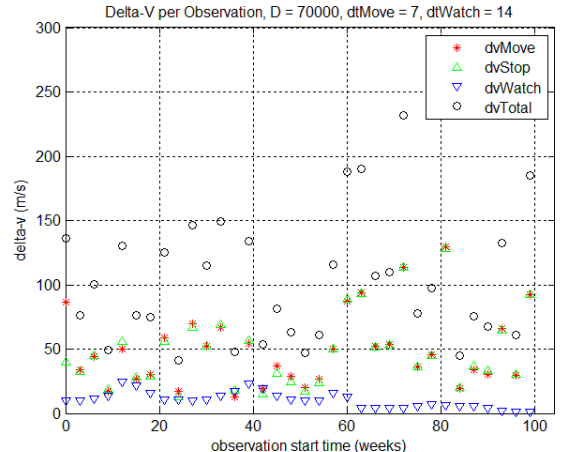

- Increase in D from 35,000 to 70,000 proportionately increases dvMove, dvStop and dvWatch

- $\quad$ Slope of delta-v vs forced displacement remains the same
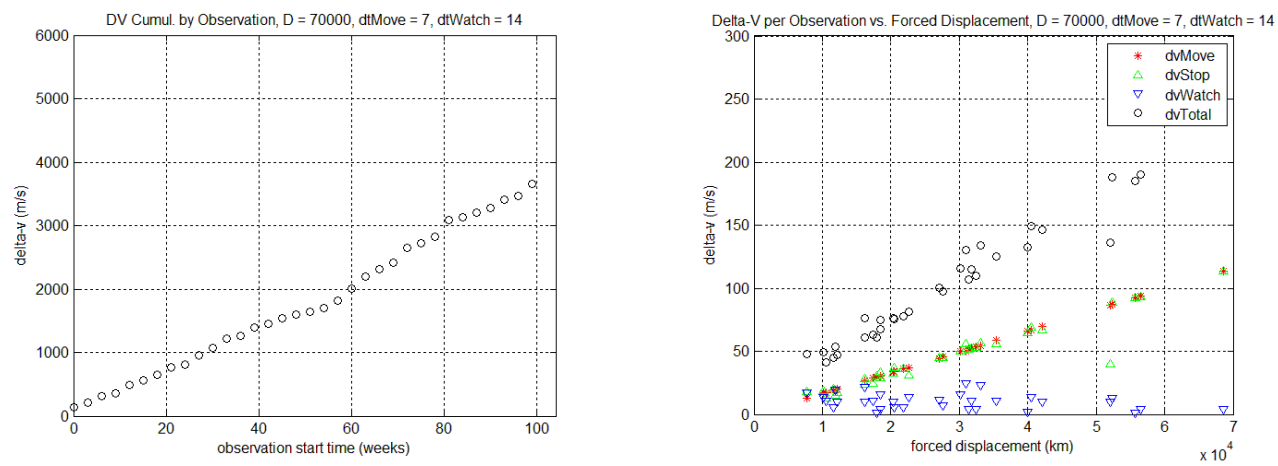
Figure 5. Effect of increasing D parameter (distance between telescope and starshade)

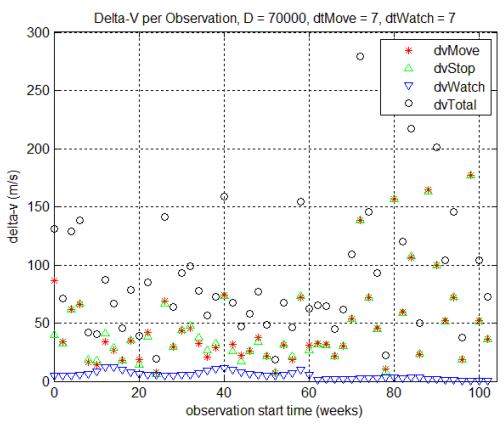

- $\quad$ Decrease in watch (observation) interval to 7 days decreases dvWatch, and does not affect the other delta-v terms, so delta-v total decreases for each observation. Since dvWatch is small, the decrease is small.

- The decrease in dtWatch increases the number of observations, and the delta-v total for the mission increases. The slope increases because the targets most easily reached have already been observed.
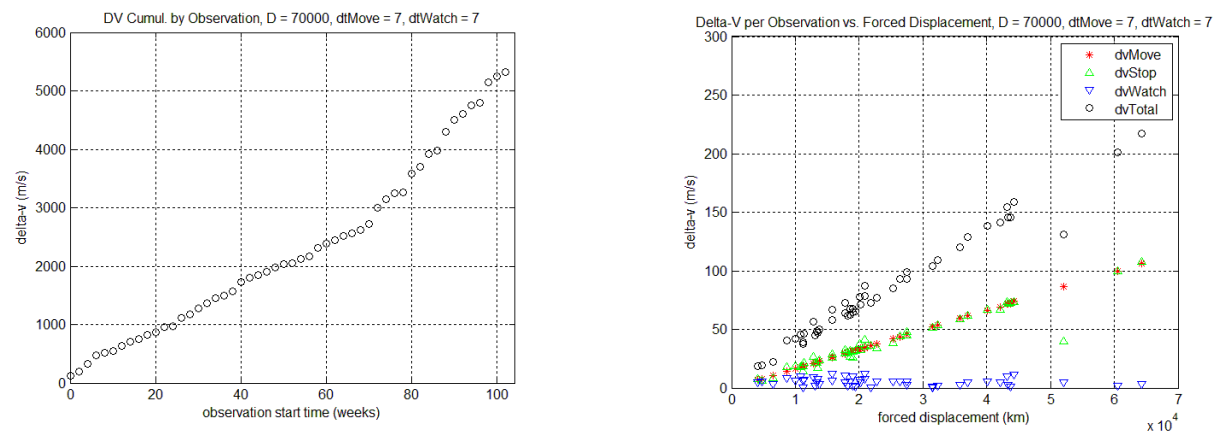

Figure 6. Effect of decreasing dtWatch parameter

\section{$D=70,000 \mathrm{~km}$, dtMove $=14$ days, dtWatch $=7$ days}

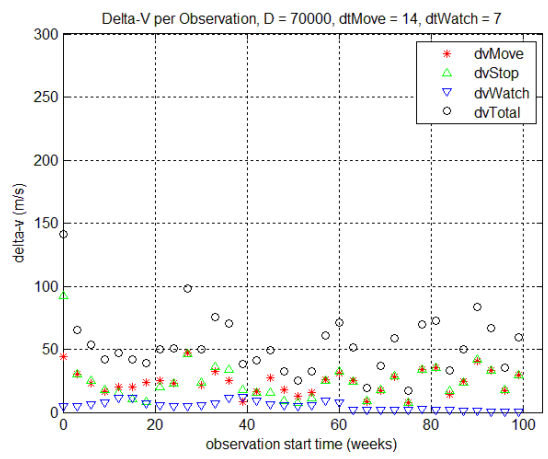

- Increasing dtMove decreases dvMove and decreases dvStop

- Decreasing dtWatch decreases dvWatch

- Hence the total delta-v per observation decreases

- The total dtMove+dtWatch is unchanged so number of observations remains the same

- When targets are selected to minimize delta$v$, average forced displacement is about $35 \%$ of telescope-occulter distance
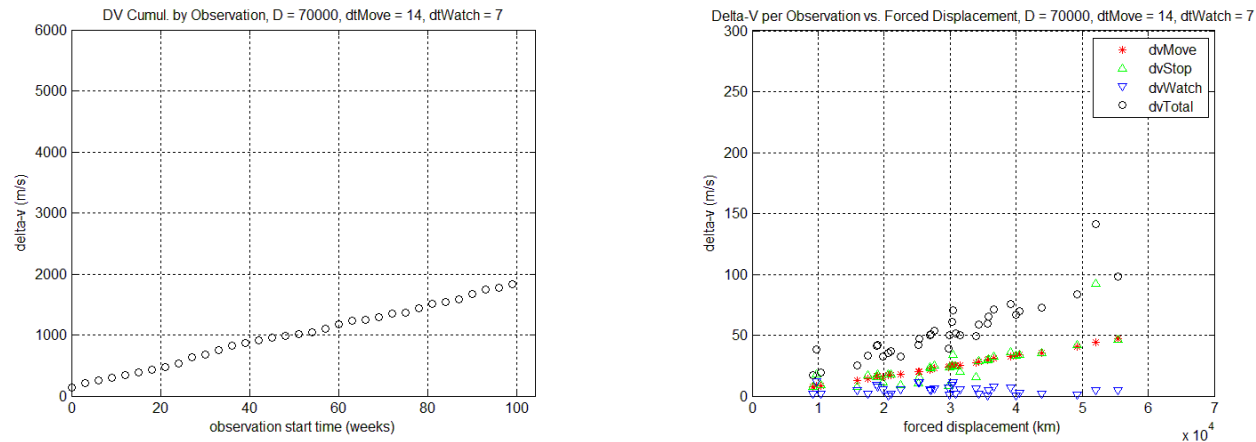
Figure 7. Effect of increasing dtMove Parameter

$D=7, C$

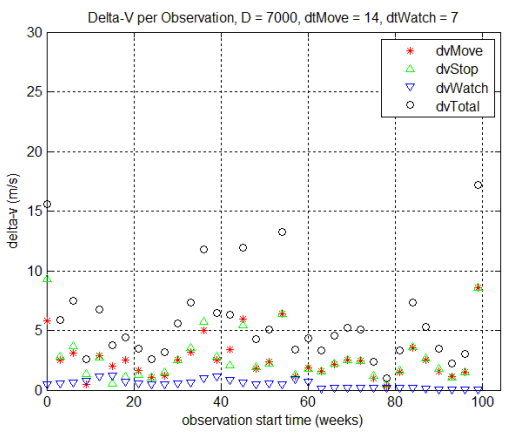

- $\quad$ Decrease D from 70,000 km to 7,000 km

- Note that delta-v scale has been decreased by a factor of 10 relative to previous plots
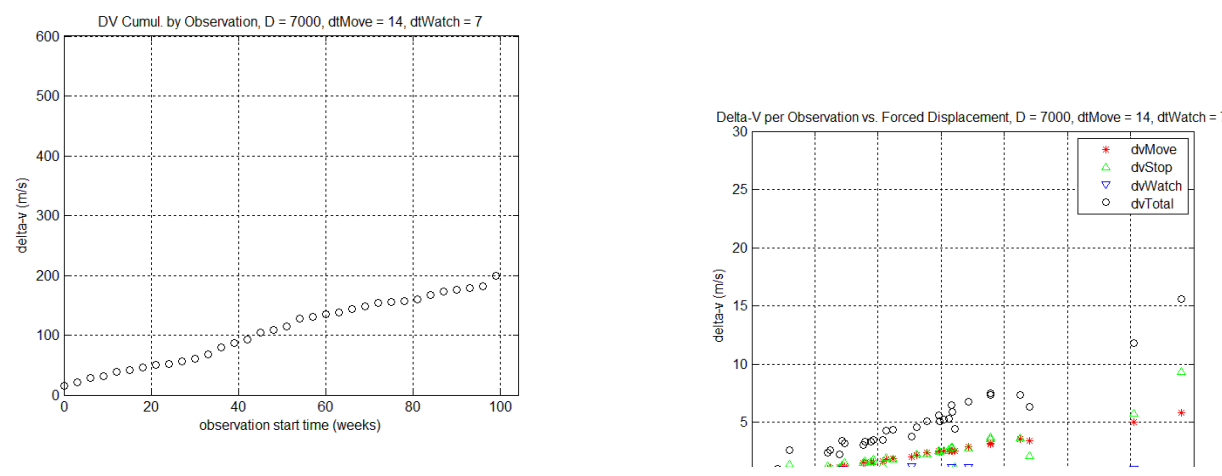

Figure 8. Effect of decreasing D parameter

\section{$D=70,000 \mathrm{~km}$, dtMove $=14$ days, dtWatch $=7$ days}

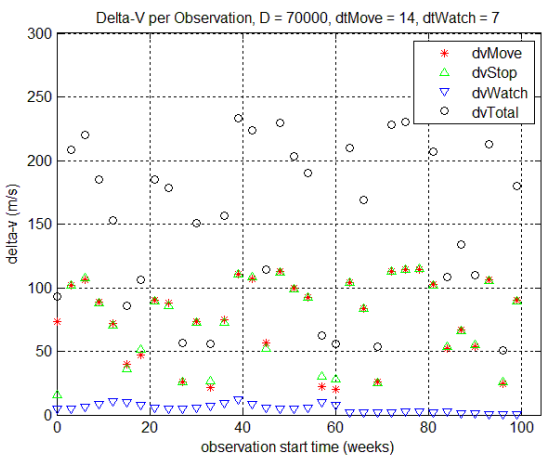

- In this case instead of choosing target with least delta-v required, we choose a random visible target

- When targets are selected randomly, average forced displacement is about $105 \%$ of telescope-occulter distance
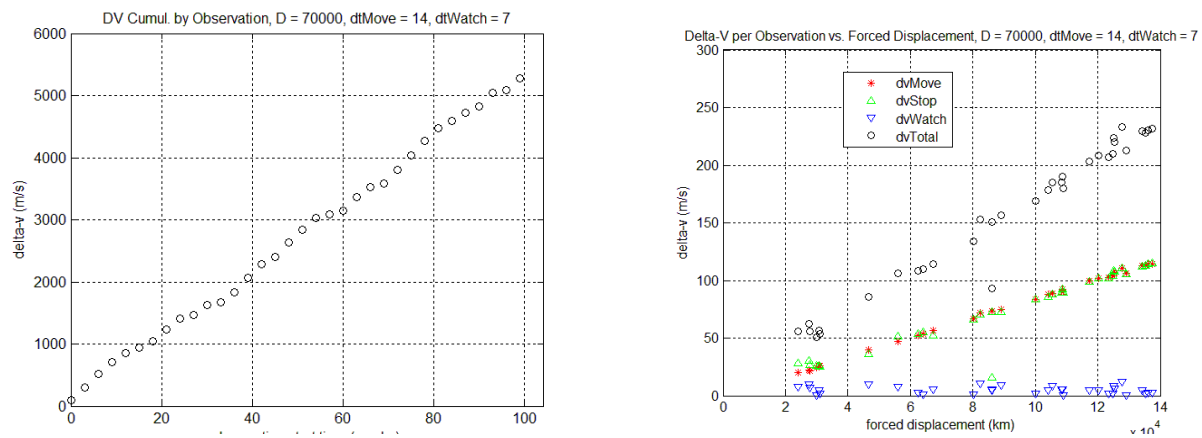
Figure 9. Effect of selecting target randomly instead of target requiring $10^{\text {th }}$ percentile delta-v as in Figure 7.



Figure 10. Angle of starshade set with respect to the sun so that SRP ballistic coefficients are nearly equal

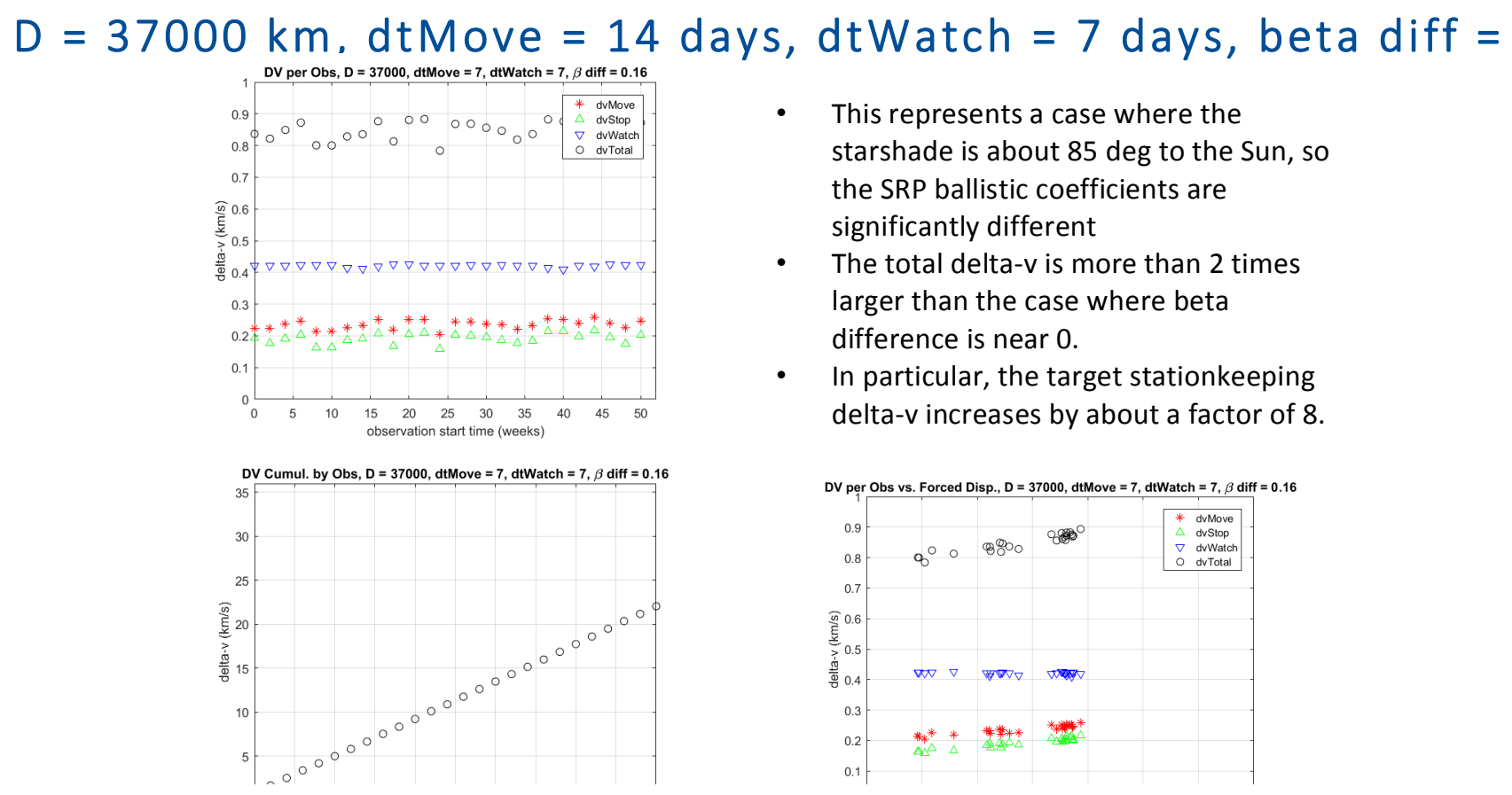


Figure 11. Angle of starshade with respect to sun readjusted, with significant change in required delta-v

\section{REFERENCES}

[1] Savransky et al., "Analyzing the Designs of Planet-Finding Missions”, PASP, Volume 122(890), pp. 401-419 (2010).

[2] Turnbull et al., "The Search for Habitable Worlds. 1. The Viability of a Starshade Mission”, PASP, Volume 124(915), pp. 418-447 (2012).

[3] Trabert et al., "Design reference missions for the exoplanet starshade (Exo-S) probe-class study", PSPIE, Volume 9605, id. 96050 Y 12 pp. (2015).

[4] Stark et al., "Maximized ExoEarth Candidate Yields for Starshades", eprint arXiv:1605.04915, (2016).

[5] Seager, et al., "Exo-S: Starshade Probe-Class Exoplanet Direct Imaging Mission Concept”, JPL, (2015). 
